\title{
THE HAHN-BANACH THEOREM FOR FINITE DIMENSIONAL SPACES
}

\author{
BY \\ WILLIAM E. BONNICE AND ROBERT J. SILVERMAN(1)
}

1. Introduction. An immediate generalization of the Hahn-Banach Theorem on the dominated extension of linear functionals is obtained by replacing the real number field by a boundedly complete vector lattice as the range space of the function. The question arises: Do boundedly complete vector lattices characterize those range spaces which permit dominated extensions? It is proved in [1], [4] that an ordered linear space is a boundedly complete vector lattice if and only if both the positive wedge is lineally closed and the Hahn-Banach Theorem holds with the space taken as the range of the extensions. Thus, the above question has an affirmative answer if the positive wedge must be closed in a space for which the Hahn-Banach Theorem is valid. This paper shows that at least for finite dimensional ordered linear spaces this is indeed the case.

An example is presented in [4] of a two dimensional ordered linear space whose positive wedge is not lineally closed and it is erroneously asserted that this space permits Hahn-Banach type extensions. The error in the argument is that the extensions described may not be well defined.

$\$ 2$ introduces preliminary definitions and theorems. In $\S 3$, finite dimensional boundedly complete vector lattices are characterized by the existence of a "partly positive basis" for the positive wedge. This characterization is a slight generalization of a theorem of Yudin [5] or Nagy [3]. In $\$ 4$ it is proved that if a finite dimensional ordered linear space permits Hahn-Banach type extensions, then the closure of the positive wedge determines an ordered linear space which is a boundedly complete vector lattice. This restricts the class of positive wedges which must be considered in ascertaining the class which admits dominated extensions.

Examples are given in $\$ 5$ which are used in $\$ 6$ to complete the proof that a finite dimensional ordered linear space admits dominated extensions if and only if the space is a boundedly complete vector lattice.

2. Preliminaries. Let $(V, C)$ be an ordered linear space (OLS) over the real number field $R$. That is, $V$ is a linear space, $C=\{v \in V \mid v \geqq 0\}$ is the positive

Received by the editors November 24, 1963 and, in revised form, August 12, 1964 and April 5, 1965.

(1) The work of both authors was supported by the National Science Foundation [NSF G-24345]. 
wedge of $V$, where ( $\geqq)$ is a transitive relation such that, if $v_{1} \geqq v_{2}$, then $t v_{1} \geqq t v_{2}$ and $v_{1}+v \geqq v_{2}+v$ for every non-negative number $t$ and all $v \in V$. The positive wedge determines the ordering: $v_{1} \geqq v_{2}$ if and only if $v_{1}-v_{2} \in C$. Conversely, if $C$ is any wedge (i.e., $C$ is a nonempty set, closed under addition and multiplication by non-negative scalars) in a vector space, then $C$ is a positive wedge relative to the ordering: $v_{1} \geqq v_{2}$ if and only if $v_{1}-v_{2} \in C$.

The wedge $C$ is lineally closed if every line intersects $C$ in a set which is closed relative to the line. The lineal closure $\bar{C}$ of a wedge $C$ is the union of closures (in the line) of all line segments contained in the wedge. Clearly, if $C$ is lineally closed, $C=\bar{C}$. A core point of a convex set is a point in the convex set such that every line through the point contains an open segment which contains the point and is contained in the set.

If $C$ is a finite dimensional wedge (i.e., the linear hull of $C$ is finite dimensional), then $\bar{C}$ coincides with the closure of $C$ relative to any topology which makes the linear hull of $C$ into a linear topological space, a core point and an interior point are synonomous, and $C$ has an interior (core) point relative to its linear hull. These statements are not generally true for infinite dimensional wedges. Further, the closure of $C$ is the union of closures of those line segments which contain the core point and are contained in $C$.

The OLS $(V, C)$ has the least upper bound property (LUBP) (or is a boundedly complete vector lattice) if every set of elements with an upper bound has a least upper bound (not necessarily unique). An OLS $(V, C)$ has the Hahn-Banach extension property (HBEP) if given (1) a real linear space $Y,(2)$ a linear subspace $X$ of $Y$, (3) a function $p: Y \rightarrow V$ which is sublinear, (i.e., $p(y)+p\left(y^{\prime}\right) \geqq p\left(y+y^{\prime}\right)$ and $\left.p(t y)=t p(y), y, y^{\prime} \in Y, t \geqq 0\right)$ and (4) a linear function $f: X \rightarrow V$ such that $p(x) \geqq f(x)$ for all $x \in X$, implies there is a linear extension $F: Y \rightarrow V$ of $f$ such that $p(y) \geqq F(y)$ for all $y \in Y$. It is proved in [1], [4] that $(V, C)$ has the LUBP if and only if $(V, C)$ has the $H B E P$ and $C$ is lineally closed.

The following three lemmas show that the HBEP, the LUBP, and lineal closure of the positive wedge are independent of the containing space. So henceforth we may speak of a wedge having the HBEP or the LUBP or being lineally closed without mention of the containing space. If $D$ is a subset of a vector space, lin $D$ will denote the linear hull of $D$.

LEMMA 2.1. The OLS $(V, C)$ has the HBEP if and only if the OLS (lin $C, C)$ has the HBEP.

Proof. Assume that $(V, C)$ has the HBEP. Let $p: Y \rightarrow$ lin $C$ be a sublinear function; $f: X \rightarrow \operatorname{lin} C$ be a linear function on the linear subspace $X$ of $Y$ such that for all $x \in X, p(x)-f(x) \in C$. Then, there exists a linear extension $F: Y \rightarrow V$ of $f$ such that for all $y \in Y, p(y)-F(y) \in C$. Thus

$$
F(y)=p(y)-(p(y)-F(y)) \in(\operatorname{lin} C)-C \subset \operatorname{lin} C .
$$


Assume (lin $C, C$ ) has the HBEP. Let $\left\{b_{\alpha} \mid \alpha \in A^{\prime}\right\} \subset C$ be an algebraic basis for lin $C$. Extend $\left\{b_{\alpha} \mid \alpha \in A^{\prime}\right\}$ to an algebraic basis $\left\{b_{\alpha} \mid \alpha \in A\right\}$ of $V$. Let $X$ be a linear subspace of linear space $Y, p: Y \rightarrow V$ be sublinear with respect to $C$, and $f: X \rightarrow V$ be linear and satisfy $p(x)-f(x) \in C$ for $x \in X$. Consider the coefficient functionals $\left\{p_{\alpha} \mid \alpha \in A\right\}$ so that $p(z)=\sum_{\alpha \in A} p_{\alpha}(z) b_{\alpha}$ for $z \in Y$, and functions $p^{\prime}$ and $p^{\prime \prime}$ defined by $p^{\prime}(z)=\Sigma_{\alpha \in A^{\prime}} p_{\alpha}(z) b_{\alpha}$ and $p^{\prime \prime}(z)=\Sigma_{\alpha \notin A^{\prime}} p_{\alpha}(z) b_{\alpha}$. The functions $p^{\prime}$ and $p^{\prime \prime}$ are well defined, positive homogeneous, and $p=p^{\prime}+p^{\prime \prime}$. Moreover, from $p\left(z_{1}\right)+p\left(z_{2}\right)-p\left(z_{1}+z_{2}\right) \in C$, it follows that $p^{\prime \prime}\left(z_{1}\right)+p^{\prime \prime}\left(z_{2}\right)-p^{\prime \prime}\left(z_{1}+z_{2}\right)=0$ and hence that $p^{\prime}\left(z_{1}\right)+p^{\prime}\left(z_{2}\right)-p^{\prime}\left(z_{1}+z_{2}\right) \in C$. Thus $p^{\prime \prime}$ is linear and $p^{\prime}$ is sublinear.

Also consider the coefficient functionals $\left\{f_{\alpha} \mid \alpha \in A\right\}$ and functions $f^{\prime}$ and $f^{\prime \prime}$ so that $f(x)=\sum_{\alpha \in A} f_{\alpha}(z) b_{\alpha}, f^{\prime}(x)=\sum_{\alpha \in A^{\prime}} f_{\alpha}(x) b_{\alpha}$ and $f^{\prime \prime}(x)=\sum_{\alpha \notin A^{\prime}} f_{\alpha}(x) b_{\alpha}$ for $x \in X$. Then $f=f^{\prime}+f^{\prime \prime}$ and $f^{\prime}$ and $f^{\prime \prime}$ are linear. Further, $p(x)-f(x) \in C$ for all $x \in X$ implies that $p^{\prime \prime}(x)=f^{\prime \prime}(x)$ and $p^{\prime}(x)-f^{\prime}(x) \in C$ for all $x \in X$. By hypothesis let $F^{\prime}: Y \rightarrow \operatorname{lin} C$ be a linear extension of $f^{\prime}$ such that $p^{\prime}(z)-F^{\prime}(z) \in C, z \in Y$. Define $F^{\prime \prime}: Y \rightarrow V$ by $F^{\prime \prime}(z)=p^{\prime \prime}(z), z \in Y$. Then $F=F^{\prime}+F^{\prime \prime}$ on $Y$ to $V$ is a linear extension of $f$ such that $p(z)-F(z) \in C, z \in Y$.

LEMMA 2.2. An $O L S(V, C)$ has the LUBP if and only if (lin $C, C)$ has the $L U B P$.

Proof. Assume $(V, C)$ has the LUBP. Let $A$ be a subset of lin $C$ which has an upper bound $b_{0} \in \operatorname{lin} C$. By assumption there is a $v_{1} \in V$ such that $v_{1}$ is a least upper bound of $A$ in $V$, hence in $\operatorname{lin} C$, if $v_{1} \in \operatorname{lin} C$. But if $a \in A$, then $v_{1}-a \in C$, and $v_{1} \in a+C \subset \operatorname{lin} C$.

Conversely, if (lin $C, C$ ) has the LUBP, let $b_{0}$ be an upper bound of a subset $A \subset V$. Then $A^{\prime}=\left\{b_{0}-a \mid a \in A\right\}$ is a subset of $C$ and hence has a greatest lower bound $b_{1} \in \operatorname{lin} C$. So $b_{2}=b_{0}-b_{1} \geqq a, a \in A$. If $u \geqq a$ for all $a \in A$, then $u-b_{0}-a+b_{0} \in C$ and therefore $-b_{0}+u \in \operatorname{lin} C$ and $b_{0}-u \leqq b_{1}$. Thus, $u \geqq b_{0}-b_{1}=b_{2}$.

LEMMA 2.3. In an $O L S(V, C), C$ is linearly closed in $V$ if and only if $C$ is linearly closed in lin $C$.

The proof is omitted.

LEMmA 2.4. If $C$ is a wedge in linear space $V$, then $\operatorname{lin} C=\operatorname{lin} \bar{C}$.

Proof. If $v \in \bar{C}$, then there is a $v_{1} \in C$ such that $\lambda v+(1-\lambda) v_{1} \in C, 0 \leqq \lambda<1$ Thus $v \in \operatorname{lin} C$ and $\operatorname{lin} C=\operatorname{lin} \bar{C}$.

An element $x$ in a wedge $C$ is sharp if $-x \notin C$. A wedge $C$ is $\operatorname{sharp}$ ( $C$ is a cone) if each nonzero element in $C$ is sharp. It is well known and easily checked that a wedge $C$ is sharp if and only if $a \leqq b \leqq a$ implies $a=b$. In this case least upper bounds are unique if they exist. 
A linear map $\phi$ from $\operatorname{OLS}(V, C)$ to $\operatorname{OLS}\left(V^{\prime}, C^{\prime}\right)$ is positive (or monotone) if $\phi(C) \subset C^{\prime}$. Two OLS $(V, C)$ and $\left(V^{\prime}, C^{\prime}\right)$ are isomorphic and $\phi: V \rightarrow V^{\prime}$ is an isomorphism if $\phi$ is linear, one to one, onto and $\phi$ and $\phi^{-1}$ are monotone. Wedges $C$ and $C^{\prime}$ are said to be isomorphic if (lin $C, C$ ) and (lin $\left.C^{\prime}, C^{\prime}\right)$ are isomorphic. Then $C$ and $C^{\prime}$ are isomorphic if and only if there exist $\operatorname{OLS}(V, C)$ and $\left(V^{\prime}, C^{\prime}\right)$ which are isomorphic. It is also readily verified that the LUBP, the HBEP, and lineal closure of the positive wedge are preserved by an isomorphism from one OLS to another.

The wedge $C$ in an OLS $(V, C)$ is reproducing if $V=$ lin $C$. Wedge $C$ is proper if $C \neq V$.

THEOREM 2.5 (Monotone Extension Theorem [1]). If $(V, K)$ is an OLS, $K$ has a core point $v_{0}$ and $X$ is a linear subspace of $V$ such that $v_{0}$ is in $X$, then any monotone linear functional defined on $X$ can be extended to a monotone linear functional defined on $V$.

Let $K$ be a wedge in linear space $V, K^{\prime}$ the wedge of positive linear functionals on $V$ and $V^{\prime}=\operatorname{lin} K^{\prime}$. Let $Q$ be the canonical map of $V$ into the conjugate space $V^{\prime \prime}$ of linear functionals on $V^{\prime}, Q v(g)=g(v), v \in V, g \in V^{\prime}$. Let

$$
\tilde{K}=\left\{v \in V \mid g(v) \geqq 0, g \in K^{\prime}\right\} .
$$

THEOREM 2.6. If $(V, K)$ is an $O L S$ with sharp reproducing wedge $K$ with core point, then (1) $\widetilde{K}=R$, (2) $K^{\prime}=R^{\prime}$, (3) $Q$ is an isomorphism from $(V, R)$ into $\left(V^{\prime \prime}, K^{\prime \prime}\right)$ (and onto if $V$ is finite dimensional).

The proof of this theorem follows from Theorem 2.5 in a direct manner and is omitted.

3. Partly positive bases. Let $V$ be an $n$-dimensional vector space, $K$ a proper, reproducing wedge in $V$. A basis $B=\left\{b_{1}, \cdots, b_{n}\right\}$ of $V$ is a $k$-partly positive basis for $K$ if and only if $B \subset K$ and there is an integer $k, 1 \leqq k \leqq n$, such that $v=\sum_{i=1}^{n} v_{i} b_{i} \in K$ if and only if $v_{i} \geqq 0, i=1,2, \cdots, k$. If $k=n$, the basis $B$ is called a positive basis for $K$.

THEOREM 3.1. (1) The wedge $K$ has the LUBP if and only if there exists a $k$-partly positive basis for $K$. (2) If $K$ has a $k$-partly positive basis, then $K$ is sharp if and only if $k=n$. (3) The number $k$ associated with a partly positive basis is unique.

Because Theorem 3.1 is a well known unpublished result, and be can proven without too much difficulty from Theorem 3.2 below of Yudin [5] or Nagy [3], the proof of 3.1 is omitted.

THEOREM 3.2. Let $K$ be a proper, reproducing, sharp wedge in a finite dimensional vector space $V$ so that $(V, K)$ is a vector lattice. Then there is a positive basis for $K$. 
THEOREM 3.3. A proper, reproducing wedge $K$ in a finite dimensional space $V$ has the LUBP if and only if $K$ is the intersection of $k$ closed halfspaces, $1 \leqq k \leqq n$ (i.e., there exists $k$ linearly independent linear functionals $g_{1}, \cdots, g_{k}$ defined on $V$ such that $v \in K$ if and only if $\left.g_{i}(v) \geqq 0, i=1, \cdots, k\right)$.

Proof. Let $B=\left\{b_{1}, \cdots, b_{n}\right\}$ be a $k$-partly positive basis for $K$. Then, as is well known, there exists linear functionals $g_{1}, \cdots, g_{n}$ such that $g_{i}\left(b_{j}\right)=\delta_{i j}, i, j=1, \cdots, n$ and if $v=\sum_{i=1}^{n} v_{i} b_{i}$, then $v_{i}=g_{i}(v)$. Thus, $g_{1}, \cdots, g_{k}$ satisfy the corollary.

The converse follows by choosing $b_{i}, i=1, \cdots, k$, so that

$$
g_{j}\left(b_{i}\right)=\delta_{j i}, \quad j=1, \cdots, k .
$$

Extend $b_{1}, \cdots, b_{k}$ to a basis $B=\left\{b_{1}, \cdots, b_{n}\right\}$ of $V$. It follows that $B$ is a $k$-partly positive basis of $K$.

4. The closure of a wedge with the HBEP. The result of this section shows that attention can be restricted to wedges whose closure has the LUBP in determining finite dimensional wedges with the HBEP.

THEOREM 4.1. Let $K$ be a finite dimensional wedge with the HBEP, then $R$, the closure of $K$, has the HBEP and thus the LUBP.

A definition and lemma are required. An OLS $(V, K)$ has the WHBEP if and only if for any quadruple $(Y, X, p, f)$ where (a) $Y$ is a linear space, (b) $X$ is a linear subspace of $Y$, (c) $p: Y \rightarrow V, p$ is sublinear with respect to $K$, (d) $f: X \rightarrow V, f$ linear, $p(x)-f(x) \in K, x \in X$, implies that there is an $F: Y \rightarrow V$, such that $F$ is a linear extension of $f$ and $p(z)-F(z) \in R, z \in Y$.

LEMMA 4.2. Let $K$ be a finite dimensional wedge such that $R$ is sharp and (lin $K, K$ ) has the WHBEP, then $R$ has the HBEP.

Proof of 4.2. (1) Let $Y$ be a vector space, $X$ a linear subspace of $Y, p: Y \rightarrow V$ such that $p$ is sublinear with respect to $R, f: X \rightarrow V$ such that $f$ is linear and $p(x)-f(x) \in R, x \in X$. Note that $V=\operatorname{lin} K$.

(2) Let $\left\{u_{\alpha}\right\}_{\alpha \in A}$ be an algebraic basis of $Y$ and $z=\sum_{\alpha \in A} \zeta_{\alpha} u_{\alpha}, z \in Y$. Define $q: Y \rightarrow R$, the real number field, by $q(z)=\left(\Sigma_{\alpha \in A}\left|\zeta_{\alpha}\right|^{2}\right)^{1 / 2}, z \in Y$. The functional is well defined, sublinear and $q\left(z_{1}+z_{2}\right)=q\left(z_{1}\right)+q\left(z_{2}\right)$ if and only if $z_{1}=\lambda z_{2}$ or $z_{2}=\lambda z_{1}$ for some real number $\lambda \geqq 0$. This is readily verified.

(3) Let $v_{0}$ be a core point of $K$. For each real number $\varepsilon>0$, define $p_{\varepsilon}: Y \rightarrow V$ by $p_{\varepsilon}(z)=p(z)+\varepsilon q(z) v_{0}$. Then $p_{\varepsilon}$ is sublinear with respect to $K$. In fact, for every $z_{1}, z_{2} \in Y,-p_{\varepsilon}\left(z_{1}+z_{2}\right)+p_{\varepsilon}\left(z_{1}\right)+p_{\varepsilon}\left(z_{2}\right)$ is a core point of $K$ or is 0 .

This follows easily from (1) and (2) and the fact that $v_{0}$ is a core point.

(4) For each $x \in X, x \neq 0, p_{\varepsilon}(x)-f(x)=(p(x)-f(x))+\varepsilon q(x) v_{0}$ is a core point of $K$ and therefore in $K$, since $\varepsilon q(x) v_{0}$ is a core point of $K$ and $p(x)-f(x) \in R$. If $x=0, p_{8}(x)-f(x)=0$. 
(5) Thus by assumption, there is a linear extension $F_{\varepsilon}: Y \rightarrow V$ of $f$ such that $p_{\varepsilon}(z)-F_{\varepsilon}(z) \in R$, for every $z \in Y$. Let $\mathscr{F}_{\varepsilon}$ be the collection of linear extensions of $f$ which are $R$-dominated by $p_{\varepsilon}$.

(6) If $\varepsilon^{\prime} \leqq \varepsilon$, then $\mathscr{F}_{\varepsilon^{\prime}} \subset \mathscr{F}_{\varepsilon}$. For $p_{\varepsilon}(z) \geqq p_{\varepsilon^{\prime}}(z) \geqq F_{\varepsilon^{\prime}}(z), z \in Y$.

(7) By virtue of Theorem 2.6 on identifying $V$ with $V^{\prime \prime}$ and $R$ with $K^{\prime \prime}$, it may be assumed that $V$ is the conjugate space of an ordered linear space $(W, C)$, so that $\bar{K}=\{v \in V \mid v(w) \geqq 0, w \in C\}$ and $C$ is reproducing and sharp.

(8) For every $w \in C, z \in Y, F_{\varepsilon} \in \mathscr{F}_{\varepsilon}$, it follows that $\left[p_{\varepsilon}(z)-F_{\varepsilon}(z)\right](w) \geqq 0$ and $\left[p_{\varepsilon}(-z)+F_{\varepsilon}(z)\right](w) \geqq 0$. If $u \in W$, then $u=w_{1}-w_{2}$, where $w_{1}, w_{2}$ are in $C$. Hence, for every $F_{\varepsilon} \in \mathscr{F}_{\varepsilon}, z \in Y, u \in W, F_{\varepsilon}(z)(u)$ is a member of the closed bounded interval, $I(u, z)=\left[-p_{\varepsilon}(-z)\left(w_{1}\right), p_{\varepsilon}(z)\left(w_{1}\right)\right]+\left[p_{\varepsilon}(z)\left(-w_{2}\right),-p_{\varepsilon}(-z)\left(-w_{2}\right)\right]$.

(9) Therefore, $\mathscr{F}_{\varepsilon} \subset \pi\{I(u, z) \mid u \in W, z \in Y\}$, the direct product of compact sets, and hence each $\mathscr{F}_{\varepsilon}$ is a subset of a compact subset of the direct product of lines relative to the direct product topology. Each set $\mathscr{F}_{\varepsilon}$ is closed in the direct product topology. For if $\phi$ is the limit point of a net $\left\{F_{\alpha}\right\}_{\alpha \in A} \subset \mathscr{F}_{\varepsilon}$, it is easily verified that $\phi$ is linear and an extension of $f$. Further, $\phi$ is $R$-dominated by $p_{\varepsilon}$. For if $w \in C, z \in Y, \eta>0$, then there is an element $F_{\alpha}$ of the net such that $\left[p_{\varepsilon}(z)-\phi(z)\right](w)=\left[p_{\varepsilon}(z)-F_{\alpha}(z)\right](w)+\left[F_{\alpha}(z)-\phi(z)\right](w) \geqq-\eta$. Thus, $p_{\varepsilon}(z)-\phi(z) \in R$, and $\mathscr{F}_{\varepsilon}$ is closed, hence, compact.

(10) By compactness, there is an $F \in \bigcap\left\{\mathscr{F}_{\varepsilon} \mid \varepsilon>0\right\}$. Then $F$ is the desired extension of $f$ for $(p(z)-F(z))(w) \geqq\left[-\varepsilon q(z) v_{0}\right](w), w \in C, z \in Y, \varepsilon>0$. Hence, $p(z)-F(z) \in R, z \in Y$. Lemma 4.2 is proved.

Proof of 4.1. (1) Let $K$ be a proper wedge with core point $v_{0}$ relative to lin $K=V$ such that $(V, K)$ has the HBEP. Let $Y$ be a vector space, $X$ a linear subspace of $Y, p: Y \rightarrow V$, a sublinear functional with respect to $R, f: X \rightarrow V$ a linear function such that $p(x)-f(x) \in R, x \in X$.

(2) Let $T=\{v \mid-v, v \in R\}$. Clearly $T$ is a linear subspace of. $V$ and $v_{0} \notin T$. Choose an algebraic basis $\beta^{\prime}$ of $T$, Extend $\beta^{\prime}$ to a basis $\beta$ of $V$ by adjoining an appropriate linearly independent set $\beta^{\prime \prime}$ and so that $v_{0} \in \beta^{\prime \prime}$.

(3) Let $S=\operatorname{lin} \beta^{\prime \prime}$ and $K_{S}=S \cap K$. The wedge $K_{S}$ is sharp and has $v_{0}$ as a core point in $S$. That $v_{0}$ is a core point is clear. Let $v,-v \in R_{S}$. Then $v \in S \cap T$, so $v=0$.

(4) The OLS $\left(S, K_{S}\right)$ has the WHBEP. For let $Y^{\prime}$ be a linear space, $X^{\prime}$ a linear subspace of $Y^{\prime}, p^{\prime}: Y \rightarrow S$ such that $p^{\prime}$ is sublinear with respect to $K_{S} ; f^{\prime}: X^{\prime} \rightarrow S$, $f^{\prime}$ linear and $p^{\prime}(x)-f^{\prime}(x) \in K_{s}, x \in X$. By assumption $(V, K)$ has the HBEP. Thus, there is a linear extension $\phi: Y^{\prime} \rightarrow V$ of $f^{\prime}$ and $p^{\prime}(z)-\phi(z) \in K, z \in Y$.

Decompose $\phi$ into $\phi(z)=\phi^{\prime}(z)+\phi^{\prime \prime}(z), z \in Y^{\prime}$, where

$$
\phi(z)=\sum_{b \in \beta} \phi_{b}(z) b, \phi^{\prime}(z)=\sum_{b \in \beta^{\prime}} \phi_{b}(z) b, \phi^{\prime \prime}(z)=\sum_{b \in \beta^{\prime \prime}} \phi_{b}(z) b .
$$

Then, $\phi^{\prime}(z) \in T, \phi^{\prime \prime}(z) \in S, z \in Y$, and $\phi^{\prime}$ and $\phi^{\prime \prime}$ are well defined linear functions on Y. Also, $p^{\prime}(z)-\phi^{\prime \prime}(z) \in S$ and $\left(p^{\prime}(z)-\phi^{\prime \prime}(z)-\phi^{\prime}(z)\right)+\phi^{\prime}(z) \in K+T \subset R$. Thus $p^{\prime}(z)-\phi^{\prime \prime}(z) \in K_{S}$ and since $\phi^{\prime \prime}$ is an extension of $f,\left(S, K_{S}\right)$ has the WHBEP. 
(5) Therefore, by Lemma $4.2,\left(S, R_{S}\right)$ has the HBEP.

(6) Represent $p(z)=p^{\prime}(z)+p^{\prime \prime}(z), \quad z \in Y$, where

$$
p^{\prime}(z)=\sum_{b \in \beta^{\prime}} p_{b}(z) b \in T, p^{\prime \prime}(z)=\sum_{b \in \beta^{\prime \prime}} p_{b}(z) b \in S
$$

are well defined functions, $p^{\prime}: Y \rightarrow T, p^{\prime \prime}: Y \rightarrow S$. Similarly, uniquely represent $f$ by $f(z)=f^{\prime}(z)+f^{\prime \prime}(z), z \in Y$, where $f^{\prime}: X \rightarrow T, f^{\prime \prime}: X \rightarrow S$.

(7) Now, $\pm\left(p^{\prime}\left(z_{1}\right)+p^{\prime}\left(z_{2}\right)-p^{\prime}\left(z_{1}+z_{2}\right)\right) \in T \subset R, z_{1}, z_{2} \in Y$. Therefore,

$$
\begin{aligned}
\left(p\left(z_{1}\right)+p\left(z_{2}\right)-p\left(z_{1}+z_{2}\right)\right)-\left(p^{\prime}\left(z_{1}\right)+p^{\prime}\left(z_{2}\right)-p^{\prime}\left(z_{1}+z_{2}\right)\right) & \\
= & p^{\prime \prime}\left(z_{1}\right)+p^{\prime \prime}\left(z_{2}\right)-p^{\prime \prime}\left(z_{1}+z_{2}\right) \in S \cap R \subset R_{S} .
\end{aligned}
$$

Thus, $p^{\prime \prime}$ is sublinear with respect to $R_{S}$. Similarly, $\pm\left(p^{\prime}(x)-f^{\prime}(x)\right) \in T \cap R, x \in X$. Hence, $p^{\prime \prime}(x)-f^{\prime \prime}(x) \in S \cap R=R_{s}$. Thus, by (5), there is a linear extension $F^{\prime \prime}: Y \rightarrow S$ of $f^{\prime \prime}$ such that $p^{\prime \prime}(z)-F^{\prime \prime}(z) \in R_{S}, z \in Y$.

(8) Extend $f^{\prime}$ linearly in any fashion to $F^{\prime}: Y \rightarrow T$ and define $F=F^{\prime}+F^{\prime \prime}$. Then, $F$ is a linear extension of $f$ and

$$
p(z)-F(z)=\left(p^{\prime}(z)-F^{\prime}(z)\right)+\left(p^{\prime \prime}(z)-F^{\prime \prime}(z)\right) \in T+T+R_{S} \subset R .
$$

Thus, $R$ has the HBEP and the LUBP. Theorem 4.1 is proved.

5. Examples. Examples are presented in this section which will be used in the next section to show that every $n$-dimensional wedge with the HBEP also has the LUBP.

\section{EXAMPLE 1.}

LEMMA 5.1. There exists a three dimensional vector space $Y$ and a two dimensional linear subspace $X$ of $Y$, with (1) a sublinear functional $p_{2}: Y \rightarrow R$, (2) linear functionals $f_{1}: X \rightarrow R, f_{2}: X \rightarrow R$, such that $p_{2}(x)-f_{1}(x) \geqq 0, p_{2}(x)$ $-f_{2}(x) \geqq 0, x \in X$ with $p_{2}(x)-f_{2}(x)=0$ if and only if $p_{2}(x)-f_{1}(x)=0$, and (3) a set $U \subset Y$ such that for any linear extension $F_{2}: Y \rightarrow R$ of $f_{2}$, if $p_{2}(z)-F_{2}(z)$ $\geqq 0, z \in Y$, then $p_{2}(u)-F_{2}(u)=0, u \in U$, and such that for any linear extension $F_{1}: Y \rightarrow R$ of $f_{1}$ there is a $u_{0} \in U$ such that $p_{2}\left(u_{0}\right)-F_{1}\left(u_{0}\right) \neq 0$. Further, by the Hahn-Banach Theorem, a linear extension $F_{2}: Y \rightarrow R$ of $f_{2}$ exists, such that $p_{2}(z)-F_{2}(z) \geqq 0, z \in Y$.

Proof $\left({ }^{2}\right)$. Let $C(-\pi / 2,3 \pi / 2)$ be the Banach space of continuous real valued functions on the closed interval $[-\pi / 2,3 \pi / 2]$, and let $X$ be the linear subspace of

(2) The referee suggested the elegant example used in the proof of Lemma 5.1. Our original example, presented here, does not require sophisticated function space arguments but the computations involved are lengthy.

Let $Y=R_{3}, X=\{(0, b, a) \mid b, a \in R\}$. Define $f_{2}: X \rightarrow R$ by $f_{2}(0, b, a)=a+b$ and

$f_{1}: X \rightarrow R$ by $f_{1}(0, b, a)=b$. Define

$p_{2}: Y \rightarrow R$ by $p_{2}(t, b, a)=|a|+b+t, t \geqq a, b \geqq 0$;

$p_{2}(t, b, a)=|a|+b+t+(a-t)^{2} /(a-t+b), a>t, b \geqq 0$;

$p_{2}(t, b, a)=|a|+t, \quad t \geqq a, b \leqq 0 ;$

$p_{2}(t, b, a)=|a|+a, \quad a \geqq t, \quad b \leqq 0$. 
$C(-\pi / 2,3 \pi / 2)$ spanned by $\phi_{1}(t)=\cos t$ and $\phi_{2}(t)=\sin (t / 3)$. Let $Y$ be the subspace of $C(-\pi / 2,3 \pi / 2)$ spanned by $X$ and the function $\phi_{3}$, where $\phi_{3}(t)=1$, if $0 \leqq t \leqq 3 \pi / 2$, and $\phi_{3}(t)=1+\sin (t / 3)$, if $-\pi / 2 \leqq t \leqq 0$. Define $f_{2}: X \rightarrow R$ by $f_{2}(\phi)=\phi(0), \phi \in X$, and $f_{1}: X \rightarrow R$ by $f_{1}(\phi)=-\phi(\pi), \phi \in X$. Further define $p_{2}: Y \rightarrow R$ by $p_{2}(\phi)=\|\phi\|, \phi \in Y$.

Then $\left\|f_{1}\right\|=\left\|f_{2}\right\|=1$ and the following three statements are equivalent for $\phi \in X$, (a) $f_{1}(\phi)=p_{2}(\phi)$, (b) $f_{2}(\phi)=p_{2}(\phi)$, (c) $\phi=\lambda \phi_{1}, \lambda \geqq 0$. Moreover from the Riesz Representation Theorem, $f_{2}$ has a unique norm preserving linear exextension $F_{2}$ defined on all of $C(-\pi / 2,3 \pi / 2)$ and hence is defined uniquely on $Y$. For if $\mu$ is a Stieltjes measure on the interval $[-\pi / 2,3 \pi / 2]$ which represents an extension of $f_{2}$ then $\mu$ is of norm 1 and satisfies

$$
\text { (i) } \int_{-\pi / 2}^{3 \pi / 2} \cos t d \mu(t)=1, \quad \text { (ii) } \int_{-\pi / 2}^{3 \pi / 2} \sin (t / 3) d \mu(t)=0 \text {. }
$$

Statement (i) implies that the support of $\mu$ contains at most the points 0 and $\pi$, while (ii) further restricts the support of $\mu$ to consist of 0 alone. Hence the extension $F_{2}$ is unique.

Let $U=\left\{\phi \mid \phi \in Y, F_{2}(\phi)=p_{2}(\phi)\right\}$. The set $U$ contains the functions $\phi_{3}$ and $\phi_{3}-\phi_{2}$. Further $p_{2}\left(\phi_{3}\right)=p_{2}\left(\phi_{3}-\phi_{2}\right)=1$. If $F_{1}$ is any linear extension to $Y$ of $f_{1}, F_{1}\left(\phi_{3}-\phi_{2}\right)=F_{1}\left(\phi_{3}\right)+\sin (\pi / 3) \neq F_{1}\left(\phi_{3}\right)$. Thus $u_{0}$ can be taken to be one of $\phi_{3}$ or $\phi_{3}-\phi_{2}$, so Lemma 5.1 is proved.

\section{EXAMPLE 2.}

LEMMA 5.2. Let $Y=R_{2}$, the vector space of ordered pairs of real numbers, $X=\{(0, a) \mid(0, a) \in Y\}$. Then there exists, (1) a sublinear functional $r_{2}: Y \rightarrow R,(2) a$ positive homogeneous functional $r_{1}: Y \rightarrow R$ such that if $r_{2}\left(z_{1}+z_{2}\right)=r_{2}\left(z_{1}\right)+r_{2}\left(z_{2}\right)$, then $r_{1}\left(z_{1}+z_{2}\right) \leqq r_{1}\left(z_{1}\right)+r_{1}\left(z_{2}\right)$, (3) linear functionals $f_{2}: X \rightarrow R, f_{1}: X \rightarrow R$ such that $r_{2}(x)-f_{2}(x) \geqq 0, x \in X$, and if $r_{2}(x)=f_{2}(x)$, then $r_{1}(x) \geqq f_{1}(x)$, and (4) $a$ set $U \subset Y$ such that if (a) $F_{2}: Y \rightarrow R$ is a linear extension of $f_{2}$ and $r_{2}(z)-F_{2}(z) \geqq 0, z \in Y$, then $F_{2}(u)=r_{2}(u), u \in U$, and (b) if $F_{1}: Y \rightarrow R$ is a linear extension of $f_{1}$, there is a sequence $\left\{u_{n}\right\} \subset U$ such that $\lim _{n \rightarrow \infty}\left(r_{1}\left(u_{n}\right)-f_{1}\left(u_{n}\right)\right)$ $=-\infty$.

Proof. Define $r_{2}: Y \rightarrow R$ by

$r_{2}(t, a)=|a|+t$, if $t \geqq 0$;

$r_{2}(t, a)=a+a t /(a-t)$, if $t<0$ and $a>0$;

$r_{2}(t, a)=-a$, if $t \leqq 0$ and $a \leqq 0$.

Define $r_{1}: Y \rightarrow R$ by

$r_{1}(t, a)=-(a t)^{1 / 2}$, if $t \geqq 0$ and $a \geqq 0$;

$r_{1}(t, a)=0$, if $a \leqq 0$ or $t \leqq 0$.

Define $f_{2}: X \rightarrow R$ by $f_{2}(0, a)=a, f_{1}: X \rightarrow R$ by $f_{1}(0, a)=0,(0, a) \in X$. 
It is clear that $r_{1}$ and $r_{2}$ are positive homogeneous. It requires detailed computations to show analytically that $r_{2}$ is subadditive. However, a sketch of the graph of $r_{2}$ makes it obvious, geometrically, that $r_{2}$ is indeed subadditive. Hence, the computations will not be presented.

Further, from the sketch of the graph of $r_{2}$, it is clear, geometrically, that $r_{2}\left(z_{1}+z_{2}\right)=r_{2}\left(z_{1}\right)+r_{2}\left(z_{2}\right)$ where $z_{i}=\left(t_{i}, a_{i}\right), i=1,2$, if and only if (a) $z_{1}=\lambda z_{2}, \lambda \geqq 0$, or (b) $t_{1} \geqq 0, a_{1} \geqq 0, t_{2} \geqq 0, a_{2} \geqq 0$, or (c) $t_{1} \geqq 0, a_{1} \leqq 0, t_{2} \geqq 0$, $a_{2} \leqq 0$, or (d) $t_{1} \leqq 0, a_{1} \leqq 0, t_{2} \leqq 0, a_{2} \leqq 0$. Simple direct computations show that in these cases $r_{1}\left(z_{1}+z_{2}\right) \leqq r_{1}\left(z_{1}\right)+r_{1}\left(z_{2}\right)$.

Consider $(0, a)=x$. Then $r_{2}(x)-f_{2}(x)=|a|-a \geqq 0$ and $=0$ if and only if $a \geqq 0$. If $a \geqq 0$, then $r_{1}(0, a)-f_{1}(0, a)=0$.

By the Hahn-Banach Theorem there exists a linear extension $F_{2}: Y \rightarrow R$ of $f_{2}$ which is dominated by $r_{2}$.

If

$$
\begin{aligned}
& \gamma_{2}=\inf \left\{r_{2}(1, a)-f_{2}(0, a) \mid a \in R\right\} \text { and } \\
& \delta_{2}=\sup \left\{-r_{2}(-1, a)+f_{2}(0, a) \mid a \in R\right\},
\end{aligned}
$$

then $\gamma_{2} \geqq F_{2}(1,0) \geqq \delta_{2}$.

Direct computation shows that $\gamma_{2}=1=r_{2}(1, a)-f_{2}(0, a), a \geqq 0$, and $\gamma_{2}=\delta_{2}$. Let $U=\{(1, a) \mid a \geqq 0\}$. The set $U$ satisfies (4) of Lemma 5.2. Then, for any linear extension $F_{1}: Y \rightarrow R$ of $f_{1}$ with

$$
F_{1}(1,0)=c, \lim _{a \rightarrow \infty}\left(r_{1}(1, a)-F_{1}(1, a)\right)=\lim _{a \rightarrow \infty}\left(-(a)^{1 / 2}-c\right)=-\infty .
$$

The lemma is proved.

6. Finite dimensional wedges with the HBEP. Every 0-dimensional wedge (0) and 1-dimensional wedge (line through 0 or closed half-line with end-point 0 ) is lineally closed and has the LUBP and the HBEP. Also, if the positive wedge of an OLS $V$ is all of $V$ then, trivially, $V$ has the HBEP, the LUBP, and is lineally closed.

For ordered linear spaces of higher dimension with proper wedges the situation is more complicated. The general result for finite dimensional wedges is Theorem 6.1 .

THEOREM 6.1. A finite dimensional wedge $K$ has the HBEP if and only if $K$ has the LUBP.

As remarked in the introduction, Theorem 6.1 follows from already known results and

THEOREM 6.2. If $K$ is a finite dimensional wedge with the HBEP then $K$ is lineally closed.

For 2-dimensional wedges there is a stronger result. 
Corollary 6.3. A 2-dimensional wedge $K$ has the HBEP if and only if $K$ is lineally closed.

The corollary follows from Theorems 6.1 and 6.2 upon the following observations. Every closed 2-dimensional wedge is isomorphic to one of the following three wedges and each of these wedges has the $\operatorname{LUBP}\left({ }^{3}\right)$.

$$
\begin{aligned}
& K_{1}=\left\{(a, b) \in R_{2} \mid a \geqq 0, b \geqq 0\right\}, \\
& K_{2}=\left\{(a, b) \in R_{2} \mid b \geqq 0\right\}, \\
& K_{3}=R_{2}, \text { where } R_{2} \text { is the linear space of ordered pairs of real numbers. }
\end{aligned}
$$

Proof of 6.2. The proof proceeds in part by induction. Nonclosed 2-dimensional wedges play a special role.

By Theorem 4.1, if the finite dimensional wedge $K$ has the HBEP then $R$ has the LUBP. Further, $K$ can be assumed to be a proper reproducing wedge in its containing OLS $V$, and can be assumed to have nonvoid interior. Moreover, by Theorem 3.1 , there is a $k$-partly positive basis $B$ of dimension $n$ for $R$, where $1 \leqq k \leqq n$.

Let $T=\{v \mid-v, v \in R\}$. It is easily verified that $T$ is a closed linear subspace of $V$. Assume $K \neq R$ and $K$ has the HBEP.

Case 1. $T=\{0\}$. There exists a positive basis $B$ for $R$ such that

$$
v=\sum_{i=1}^{n} v_{i} b_{i} \in R \Leftrightarrow v_{i} \geqq 0, i=1, \cdots, n .
$$

Since $K$ is not closed, there is some $b^{\prime}$, say $b_{1}$, in $B$ such that $b_{1}$ is not in $K$. Let $Y, X, p_{2}, f_{2}, f_{1}$ be as in Lemma 5.1, Example 1, $\$ 5$. Define:

$$
\begin{aligned}
& p: Y \rightarrow V, p(z)=p_{2}(z) \sum_{i=1}^{n} b_{i}, \quad z \in Y ; \\
& f: X \rightarrow V, f(x)=f_{1}(x) b_{1}+f_{2}(x) \sum_{i=2}^{n} b_{i}, \quad x \in X .
\end{aligned}
$$

The function $p$ is sublinear with respect to $K$, for $v_{0}=\sum_{i=1}^{n} b_{i}$ is an interior point of $K$ and $p_{2}$ is a sublinear functional. Also, $p(x)-f(x) \in K, x \in X$, for $p(x)-f(x)=\left(p_{2}(x)-f_{1}(x)\right) b_{1}+\left(p_{2}(x)-f_{2}(x)\right) \sum_{i=2}^{n} b_{i}$. By Lemma 5.1, $p_{2}(x)-f_{1}(x) \geqq 0, \quad p_{2}(x)-f_{2}(x) \geqq 0$, and $p_{2}(x)=f_{1}(x)$ if and only if $p_{2}(x)$ $=f_{2}(x), x \in X$. Also, $\sum_{i=1}^{n} a_{i} b_{i}$ is in the interior of $K$ if $a_{i}>0, i=1, \cdots, n$.

(3) It is also easily verified that every other 2-dimensional wedge is isomorphic to one of

$C_{1}=\left\{(a, b) \in R_{2} \mid a>0, \quad b>0, \quad\right.$ or $\left.a=0, b=0\right\}$,

$C_{2}=\left\{(a, b) \in R_{2} \mid a \geqq 0, b>0, \quad\right.$ or $\left.a=0, b=0\right\}$,

$C_{3}=\left\{(a, b) \in R_{2} \mid b>0, \quad\right.$ or $\left.a=0, b=0\right\}$,

$C_{4}=\left\{(a, b) \in R_{2} \mid b>0, \quad\right.$ or $\left.a \geqq 0, \quad b=0\right\}$.

The wedge $C_{4}$ determines a lattice, but this conditon is not sufficent to guarantee that $C_{4}$ has the HBEP. 
There exists no linear extension $F: Y \rightarrow V$ of $f$ so that $p(z)-F(z) \in K, z \in Y$. For assume $F$ is a linear extension of $f, F(z)=F_{1}(z) b_{1}+\sum_{i=2}^{n} F_{2_{i}}(z) b_{i}$. Then the coefficient functions are linear, $F_{1}$ is an extension of $f_{1}$ and $F_{2_{i}}$ is an extension of $f_{2}, i=2, \cdots, n$. Further assume that $p(z)-F(z) \in K$, then $p_{2}(z)-F_{2_{i}}(z) \geqq 0$, $i=2, \cdots, n, z \in Y$. By Lemma 5.1, there is a set $U \subset Y$, so that $p_{2}(u)-F_{2_{i}}(u)=0$, $u \in U, i=2, \cdots, n$ and there is a $u_{0} \in U$ so that $p_{2}\left(u_{0}\right)-F_{1}\left(u_{0}\right) \neq 0$. Thus, $p\left(u_{0}\right)-F\left(u_{0}\right)=\left(p_{2}\left(u_{0}\right)-F_{1}\left(u_{0}\right)\right) b_{1} \in K$. This is a contradiction since $-b_{1}, b_{1} \notin K$ and $p_{2}\left(u_{0}\right)-F_{1}\left(u_{0}\right) \neq 0$.

Case 2. $T \neq\{0\}$. There are several subcases.

Case (2i) $T \cap K=\{0\}$. In this situation if $\sum_{i=1}^{n} v_{i} b_{i}=v \in K$ and $v_{i}=0$, $i=1, \cdots, k$, then $v_{i}=0, i=k+1, \cdots, n$. Again let $Y, X, p_{2}, f_{1}, f_{2}$, be as in Lemma 5.1. Define $p: Y \rightarrow V$ by $p(z)=p_{2}(z) \sum_{i=1}^{n} b_{i}, z \in Y$. As in Case $1, p$ is sublinear with respect to $K$. Define $f: X \rightarrow V, f(x)=f_{2}(x)\left(\sum_{i=1}^{k} b_{i}\right)+f_{1}(x)\left(\sum_{i=k+1}^{n} b_{i}\right), x \in X$. Again it is easily verified that $p(x)-f(x) \in K, x \in X$. Assume $F: Y \rightarrow V$ is a linear extension of $f, F(z)=\sum_{i=1}^{k} F_{2_{i}}(z) b_{i}+\sum_{i=k+1}^{n} F_{1_{i}}(z) b_{i}, z \in Y$. Thus, $p(z)-F(x) \in K$ implies that $p_{2}(z)-F_{2_{i}}(z) \geqq 0, z \in Y, i=1, \cdots, k$. Again from Lemma 5.1, choose $u_{0} \in U$, so that $p_{1}\left(u_{0}\right)-F_{1}\left(u_{0}\right) \neq 0$. Thus an extension is not possible and $K$ does not have the HBEP.

Case (2 ii) Assume $T \subset K$. Again let $Y, X, p_{2}, f_{1}, f_{2}$ be as in Lemma 5.1. Again define $p: Y \rightarrow V$ by $p(z)=p_{2}(z) \sum_{i=1}^{n} b_{i}, z \in Y$. Since $T \subset K$ there is a $b_{j} \in B, 1 \leqq j \leqq k$, say $j=1$, so that $b_{1} \notin K$. Define $f: X \rightarrow V, f(x)=f_{1}(x) b_{1}$ $+f_{2}(x) \sum_{i=2}^{n} b_{i}$. Again it is easily verified that $p$ is sublinear with respect to $K$ and $p(x)-f(x) \in K, x \in X$. Assume $F: Y \rightarrow V$ is a linear extension of $f$ such that $p(z)-F(z) \in K, z \in Y$, and $F(z)=F_{1}(z) b_{1}+\sum_{1=2}^{n} F_{2_{i}}(z) b_{i}$. Choosing $u_{0} \in U$ so that $p_{2}\left(u_{0}\right)-F_{1}\left(u_{0}\right) \neq 0$, then one has clearly, $\left(p_{2}\left(u_{0}\right)-F_{1}\left(u_{0}\right)\right) b_{1} \in K$, since $\pm \sum_{i=k+1}^{n}\left(p_{2}\left(u_{0}\right)-F_{2_{i}}\left(u_{0}\right)\right) b_{i} \in T$ and $p_{2}\left(u_{0}\right)=F_{2_{i}}\left(u_{0}\right), i=2, \cdots, k$. Thus, a contradiction is obtained in this case and $K$ must be closed.

Case (2 iii) Suppose $T \cap K$ is not closed. Then by induction on dimension, $K$ does not have the HBEP, for $T \cap K$ has dimension less than that of $K$ and it will be shown that:

If $K$ has the HBEP, then $T \cap K$ has the HBEP.

Let $Y$ be a linear space, $X$ a linear subspace of $Y, p: Y \rightarrow V$ a function sublinear with respect to $T \cap K, f: X \rightarrow V$ a linear function such that $p(x)-f(x) \in T \cap K$, $x \in X$. Then there is a linear extension $F: Y \rightarrow V$ of $f$ such that $p(z)-F(z) \in K$, $z \in Y$. Thus, $p( \pm z)-F( \pm z)=\sum_{i=1}^{k}-F_{i}( \pm z) b_{i}+\sum_{i=k+1}^{n}\left(p_{i}( \pm z)-F_{i}( \pm z)\right) b_{i}$ $z \in Y$, where the $p_{i}$ 's and $F_{i}$ 's are the coefficient functions of $p$ and $F$ respectively. The functions $F_{i}$ are linear. Also if $v=\sum_{i=1}^{n} a_{i} b_{i} \in K$, then $a_{i} \geqq 0, i=1, \cdots, k$. Thus, $F_{i}=0, i=1, \cdots, k$ and $F(z) \in T, z \in Y$, and $p(z)-F(z) \in T \cap K, z \in Y$.

Case (2iv). Suppose $T \cap K$ is closed, $T \notin K, T \cap K \neq\{0\}$, and for every $b \in B$, if $b \in T \cap K$ then $-b \in T \cap K$. It may be assumed that $b_{n} \in B \cap T \cap K$. 
Let $B^{\prime}=\left\{b \mid b \in B, b \neq b_{n}\right\}$ and let $K^{\prime}=K \cap \operatorname{lin} B^{\prime}$. Then $K^{\prime}$ is not closed since $T \notin K$. Further, $K^{\prime}$ has dimension less than $K$. Then by induction and the following lemma, $K$ does not have the HBEP. The wedge $K^{\prime}$ has the HBEP if $K$ has the HBEP.

Let $Y$ be a linear space, and $X$ be a linear subspace of $Y$. Let $p: Y \rightarrow V$ be a sublinear function with respect to $K^{\prime}$ and $p(z)=\sum_{i=1}^{n-1} p_{i}(z) b_{i}$. Let $f: X \rightarrow V, f$ linear, be so that $f(x)=\sum_{i=1}^{-1} f_{i}(x) b_{i}$ and $p(x)-f(x) \in K^{\prime}, x \in X$.

Then there is a linear extension $G: Y \rightarrow V$ of $f, G(z)=\sum_{i=1}^{n} G_{i}(z) b_{i}$ and $p(z)-G(z) \in K, \quad z \in Y$. Thus, $p(z)-G(z)-G_{n}(z) b_{n} \in K \cap \operatorname{lin} B^{\prime}=K^{\prime}, \quad z \in Y$. Letting $F(z)=G(z)-G_{n}(z) b_{n}, z \in Y$, then $F$ is the desired extension and $K^{\prime}$ has the HBEP.

Case (2v). Suppose $T \cap K$ is closed, $T \notin K, T \cap K \neq\{0\}$ and there is a $b \in B$, say $b_{n}$, so that $b_{n} \in T \cap K$ and $-b_{n} \notin T \cap K$. This case breaks down into 2 subcases.

If $n=2$, the only wedge which need be considered is $C_{4}=\left\{(a, b) \in R_{2} \mid b>0\right.$, or $a \geqq 0, b=0\}$. (See comment at end of Footnote 3.)

Let $Y$ be a linear space, $X$ a linear subspace of $Y$. Then, $q: Y \rightarrow R_{2}$, $q(z)=\left(q_{1}(z), q_{2}(z)\right), z \in Y$, is sublinear with respect to $C_{4}$ if and only if $q_{2}$ is a sublinear functional, $q_{1}$ is positive homogeneous, and if $q_{2}\left(z_{1}\right)+q_{2}\left(z_{2}\right)$ $=q_{2}\left(z_{1}+z_{2}\right)$, then $q_{1}\left(z_{1}\right)+q_{1}\left(z_{2}\right) \geqq q_{1}\left(z_{1}+z_{2}\right)$.

Let $f: X \rightarrow R_{2}, f(x)=\left(f_{1}(x), f_{2}(x)\right), x \in X, f$ linear. Then $f_{1}$ and $f_{2}$ are linear functionals. For every $x \in X, q(x)-f(x) \in C_{4}$ if and only if $q_{2}(x)-f_{2}(x) \geqq 0$, and if $q_{2}(x)-f_{2}(x)=0$, then $q_{1}(x)-f_{1}(x) \geqq 0$. Further, if $F: Y \rightarrow R_{2}, F(z)=\left(F_{1}(z)\right.$, $\left.F_{2}(z)\right), z \in Y$, is a linear extension of $f$, then $F_{1}$ is a linear extension of $f_{1}$ and $F_{2}$ is a linear extension of $f_{2}$. Also, if $q(z)-F(z) \in C_{4}, z \in Y$, then $q(z)-F(z)$ must satisfy the same conditions as $q(x)-f(x)$ on replacing $x$ by $z$ and $f$ by $F$.

Let $Y, X, r_{1}, r_{2}, f_{1}, f_{2}$ be as in $\S 5$, Example 2, Lemma 5.2, and $q_{1}=r_{1}, q_{2}=r_{2}$, $f(x)=\left(f_{1}(x), f_{2}(x)\right), x \in X$. Then by Lemma $5.2, q$ is sublinear with respect to $C_{4}, q(x)-f(x) \in C_{4}, x \in X$; but by Lemma 5.2 no linear extension $F: Y \rightarrow R_{2}$ of $f$ satisfies the condition $q(z)-F(z) \in C_{4}, z \in Y$.

If $n>2$, let $B^{\prime}=\left\{b \in B \mid b \neq b_{1}\right\}$ and let $K^{\prime}=K \cap \operatorname{lin} B^{\prime}$. Then clearly, $K^{\prime}$ has dimension less than $K$. Also $K^{\prime}$ is not closed since $T \notin K$ and the dimension $n$ is greater than 2 . Then by induction and the following lemma, $K$ does not have the HBEP. The wedge $K^{\prime}$ has the HBEP if $K$ has the HBEP.

Let $Y$ be a linear space; $X$ a linear subspace of $Y ; p: Y \rightarrow V$ a function sublinear with respect to $K^{\prime}$, so that $p(z)=\sum_{i=2}^{n} p_{i}(z) b_{i} ; f: X \rightarrow V, f$ linear, so that $f(x)=\sum_{i=2}^{n} f_{i}(x) b_{i}$; and $p(x)-f(x) \in K^{\prime}, x \in X$. Then there is a linear extension $F: Y \rightarrow V$ of $f, F(z)=\sum_{i=1}^{n} F_{i}(z) b_{i}$, and $p(z)-F(z) \in K, z \in Y$. Thus, $p( \pm z)-F( \pm z)=-F_{1}( \pm z) b_{1}+\sum_{i=2}^{n}\left(p_{i}( \pm z)-F_{i}( \pm z)\right) b_{i}$. Since $F_{1}$ is linear and $v=\sum_{i=1}^{n} v_{i} b_{i} \in K$ implies $v_{1} \geqq 0, F_{1}=0$. Thus $F(z) \in K^{\prime}$ and $p(z)$ $-F(z) \in K^{\prime}, z \in Y$. Theorems 6.1 and 6.2 are proved. 


\section{BIBLIOGRAPHY}

1. M. M. Day, Normed linear spaces, Academic Press, New York, 1962.

2. M. G. Krein and M. A. Rutman, Linear operators leaving invariant a cone in a Banach space, Amer. Math. Soc. Transl. No. 26, 1950.

3. B. Sz.-Nagy, Sur les lattis linéaires de dimension finie, Comment. Math. Helv. 17 (1945), 209-213.

4. R. J. Silverman and Ti Yen, The Hahn-Banach theorem and the least upper bound property, Trans. Amer. Math. Soc. 90 (1959), 523-526.

5. A. Yudin, Solution des deux problèmes de la théorie des espaces semi-ordonnés, C. R. (Dokl.) Acad. Sci. URSS (N.S.) 23 (1939), 418-422.

\section{UNIVERSITY OF NEW HAMPSHIRE, \\ DURHam, New HampshiRe}

